\title{
Eficiência de fungos filamentosos no controle biológico da vassoura de bruxa do cacaueiro
}

\author{
Moabe Mattos Oliveira ${ }^{1^{*}}$, Fábia Giovana do Val de Assis ${ }^{2, a}$ \& Patrícia Lopes Leal ${ }^{1, b}$ \\ ${ }^{1}$ Instituto Multidisciplinar em Saúde, Universidade Federal da Bahia - Campus Anísio Teixeira, Vitória da \\ Conquista, Bahia, Brasil. \\ ${ }^{2}$ Departamento de Microbiologia, Universidade Federal de Viçosa, Minas Gerais, Brasil.
}

\begin{abstract}
Resumo - Nós analisamos a eficiência de fungos no controle biológico da vassoura de bruxa em diferentes condições experimentais (laboratório, casa de vegetação e campo) a partir de uma revisão sistemática da literatura. As espécies/isolados foram divididos em três grupos e a eficiência média desses micro-organismos foi avaliada. Os grupos são representados pelos gêneros Trichoderma (eficiência de $51,5 \%)$ e Clonostachys (38\%), e pelos fungos endofíticos (22\%). No entanto, as condições experimentais podem influenciar a eficiência do controle biológico. Trichoderma e Clonostachys são os gêneros mais eficientes na redução da incidência da vassoura de bruxa e também os mais utilizados no biocontrole desta fitopatogenicidade.
\end{abstract}

Palavras-chave adicionais: biocontrole, cacau, Clonostachys, condições experimentais, micoparasitismo, Trichoderma.

\begin{abstract}
Efficiency of filamentous fungi in the biological control of witches' broom disease of cocoa) - We analyzed the fungi efficiency in the biological control of witches' broom disease in different experimental conditions (laboratory, greenhouse, and field) through a systematic review of the literature. Species/isolates were divided into three groups and the mean efficiency of these microorganisms was assessed. The groups were represented by the genera Trichoderma ( $51.5 \%$ efficiency) and Clonostachys (38\%), and the endophytic fungi (22\%). Nevertheless, experimental conditions may affect the biological control efficiency. Trichoderma and Clonostachys were the most efficient genera in reducing the incidence of witches' broom disease and also the most used to biocontrol this phytopathogenicity.

Additional key words: biocontrol, cacao, Clonostachys, experimental conditions, mycoparasitism, Trichoderma.
\end{abstract}

Perdas significativas da produção de cacau (Theobroma cacao L.) têm sido registradas nas últimas décadas no Brasil. Essas perdas são atribuídas principalmente à vassoura de bruxa, doença causada pelo fungo Moniliophthora perniciosa (Stahel) Singer (Meinhardt et al. 2008). A infecção por este fungo fitopatogênico ocorre principalmente em tecidos jovens, meristemáticos, como brotos vegetativos, almofadas florais e frutos, provocando sintomas característicos de desequilíbrio hormonal que ocorrem em interações patógeno-hospedeiro (Alves et al. 2006). Os sinais mais evidentes da doença são a formação dos brotos hipertrofiados, desenvolvimento excessivo nas regiões terminais da planta, aparecimento de inúmeras ramificações com entrenó curto e folhas geralmente grandes, curvadas ou retorcidas, aparentando vassouras (Simões 2010). Muitas técnicas têm sido adotadas para o manejo da vassoura de bruxa, como a poda fitossanitária dos tecidos infectados, aplicação de fungicidas e uso de genótipos resistentes. Esta última técnica foi considerada por Costa et al. (2009) como uma medida duradoura mas não definitiva, pois novos patótipos do fungo podem surgir, exigindo $\mathrm{o}$ desenvolvimento de novas variedades resistentes.

O prejuízo econômico causado pela vassoura de bruxa estimula a busca de métodos alternativos para $\mathrm{o}$ seu controle e o controle biológico apresenta um custo

\footnotetext{
*Autor para correspondência: moabe.10@hotmail.com;

afabi_zoo@yahoo.com.br; blealpat@yahoo.com.br

Editor responsável: Luis Fernando Pascholati Gusmão

Submetido: 30 mar. 2014; aceito: 7 jul. 2014

Publicação eletrônica: 7 nov. 2014; versão final: 23 dez. 2014
}

mais baixo e é mais seguro ao meio ambiente do que o controle químico (Bastos 2007). Vários organismos com potencial para o controle da vassoura de bruxa têm sido identificados (Souza et al. 2006) e os fungos epifíticos e endofíticos têm merecido especial atenção (Schrank et al. 2001; Samuels 2006). Dentre esses fungos, destacam-se o Trichoderma stromaticum Samuels \& Pardo-Schultheiss (Santos 2005; Souza et al. 2006; Loguercio et al. 2009), Trichoderma spp. (Simões 2010; Simões et al. 2011; Bastos 2012), Clonostachys spp. (Krauss \& Soberanis 2001; Bastos 2004, 2011) e alguns fungos endofíticos (Rubini et al. 2005; Bastos 2007; Mejía et al. 2008).

No Brasil, a comercialização de compostos a base de micro-organismos para o biocontrole já é uma realidade para produtores de cacau, sendo recomendados como parte do manejo integrado da vassoura de bruxa (Bastos 2008). No entanto, a literatura mostra resultados conflitantes sobre a eficiência desse biocontrole, o que dificulta sua aceitação mais ampla. Interações entre plantas, patógenos, microambiente e agentes de biocontrole, além das condições experimentais, podem influenciar no sucesso do biocontrole e gerar resultados conflitantes quanto a sua eficiência (Campos et al. 2009).

A fim de entender melhor a eficiência do biocontrole no combate à vassoura de bruxa, nós realizamos uma revisão sistemática sobre o assunto. Foram analisados bancos de dados sobre espécies de fungos filamentosos e endofíticos com potencial aplicação no controle biológico da vassoura de bruxa do cacaueiro. Em seguida, analisamos estatisticamente 
a eficiência desses fungos no biocontrole, avaliando os benefícios deste uso, as situações em que a aplicação do antagonista é necessária e quais os aspectos que ainda devem ser explorados.

\section{MATERIAL E Métodos}

Revisão sistemática. Em dezembro de 2013, foi realizado um levantamento de trabalhos abordando o emprego de espécies de fungos filamentosos e endofíticos no controle biológico de Moniliophthora perniciosa. Foram consultadas bibliotecas virtuais (PubMed, Scielo, CAPES e LILACS) e revistas científicas utilizando palavras-chave como "controle biológico", "cacau" e "vassoura de bruxa". Foram incluídos estudos entre os anos de 2001 e 2012 com informações sobre: 1- a eficiência relativa de fungos no controle biológico; 2- o potencial antagônico do fungo à vassoura de bruxa; ou 3- o potencial de crescimento do fungo sobre a fitopatogenicidade ou potencial de esporulação do fungo. Não foram considerados trabalhos que não apresentaram tais informações. Também não foram consideradas revisões bibliográficas ou trabalhos que não representassem fontes primárias de dados (Borges et al. 2012).

A seleção dos trabalhos foi realizada a partir de uma avaliação inicial com base no título e no resumo. Posteriormente, as variáveis de cada estudo (isolado microbiano, condições experimentais, número de amostras testadas) foram arquivadas conforme proposto por Pelozatto \& Fernandes (2011). Para eficiência de fungos endofíticos, as espécies fúngicas foram avaliadas de forma agrupada no trabalho original (Rubini et al. 2005); ou seja, estes fungos não foram analisados individualmente quanto à eficiência do biocontrole da vassoura de bruxa.

Eficiência no biocontrole. Os dados dos trabalhos selecionados foram filtrados em três etapas. A primeira procurou assegurar a coerência da publicação com os objetivos desta revisão. Em seguida, os dados foram transferidos para uma base de dados, considerando o conjunto das características gerais e das relações da base, se a publicação não apresentava um comportamento anormal. O efeito das espécies de fungos utilizadas no biocontrole foi quantificado através de sua eficiência (Ef), definida no presente trabalho como potencial de controle biológico da fitopatogenicidade de cada espécie:

$$
\mathrm{Ef}=\frac{\% \mathrm{PA}+\% \mathrm{PC}+\% \mathrm{PE}}{3}
$$

onde \%PA é o potencial antagônico à vassoura de bruxa, \%PC é o potencial de crescimento do fungo sobre a fitopatogenicidade e \%PE é o potencial de esporulação do fungo. Os micro-organismos utilizados no biocontrole foram categorizados de acordo com o valor percentual de inibição da doença, conforme sugerido por Linhares et al. (1995): eficientes (inibição de 81-100\%); moderadamente eficientes (51-80\%); pouco eficientes $(25-50 \%)$; e ineficientes $(<25 \%)$. Para avaliar a influência das condições experimentais (laboratório, casa de vegetação e campo) sobre a eficiência dos fungos no biocontrole da vassoura de bruxa, os dados foram analisados de forma independente, ou seja, espécies fúngicas por condição experimental.

Análise estatística. A eficiência das espécies de fungos no biocontrole da vassoura de bruxa foi analisada através de estatística descritiva. Avaliou-se a média e o desvio padrão da eficiência de cada espécie. O teste estatístico de Shapiro-Wilk verificou que os dados não seguem uma distribuição normal, sendo então utilizado o teste não-paramétrico de KruskalWallis para calcular a significância estatística $(\mathrm{p}<$ $0,05)$ entre as espécies e as condições dos experimentos. Esses testes foram realizados no Graph Pad Prism 5.00 (GraphPad Software Ic., San Diego, CA, EUA).

\section{RESUlTADOS}

Foram selecionados 13 estudos para essa revisão sobre eficiência de fungos no controle biológico da vassoura de bruxa, sete conduzidos no Brasil, cinco nos Estados Unidos e um na Costa Rica (Tabela 1). A Tabela 2 mostra que as maiores taxas de eficiência no controle biológico foram registradas para os isolados $T$. harzianumi Rifai (Th911) (91,86 e 90,33\%) e $T$. stromaticum $(80,90 \%)$. No entanto, percentuais de eficiência bem inferiores foram observadas para $T$. brevicompactum G.F. Kraus, C.P. Kubicek \& W. Gams (12\%), Clonostachys sp (16,70\%) e fungos endofíticos $(16,22 \%)$.

Espécies/isolados de fungos foram divididos em três grupos com base na eficiência do controle biológico: Grupo 1, contendo os fungos do gênero Trichoderma; Grupo 2, contendo fungos do gênero Clonostachys; e Grupo 3, contendo os fungos endofíticos, que não foram inseridos em nenhum dos grupos anteriores. A maior eficiência média foi observada para Trichoderma $(51,50 \%)$, seguida por Clonostachys (38\%) e pelos fungos endofíticos (22\%). Diferença significativa $(\mathrm{p}=0.0024)$ foi registrada apenas entre as eficiências do gênero Trichoderma e dos fungos endofíticos (Figura 1).

Segundo a classificação proposta por Linhares et al. (1995), apenas alguns isolados de Trichoderma foram eficientes no controle biológico da vassoura de bruxa, correspondendo a 4,8\% das espécies testadas; 46,23\% das espécies são moderadamente eficientes, 40,81\% pouco eficientes e $8,16 \%$ ineficientes.

A maior eficiência dos fungos no controle biológico da vassoura de bruxa ocorre em laboratório (média $=52,56 \%$ ), 
Tabela 1. Trabalhos selecionados para a revisão sistemática sobre a eficiência dos fungos no controle da vassoura de bruxa do cacaueiro indicando o país onde foi realizado, número de amostras selecionadas e condições experimentais.

\begin{tabular}{|c|c|c|c|}
\hline Referência & País & Número de amostras & Condições experimentais \\
\hline Krauss et al. $(2001)^{a}$ & Costa Rica & 20 & Campo \\
\hline Bastos $(2004)^{b}$ & Brasil & 20 & Casa de vegetação \\
\hline Rubini et al. $(2005)^{\mathrm{c}}$ & Estados Unidos & 5 & Casa de vegetação \\
\hline Santos $(2005)^{d}$ & Brasil & 5 & Campo \\
\hline Carvalho $(2006)^{\mathrm{e}}$ & Brasil & 21 & Campo \\
\hline Souza et al. $(2006)^{\mathrm{f}}$ & Estados Unidos & 4 & Campo \\
\hline Bastos $(2007)^{\mathrm{g}}$ & Brasil & 10 & Casa de vegetação \\
\hline Mejía et al. $(2008)^{\mathrm{h}}$ & Estados Unidos & 20 & Casa de vegetação \\
\hline Loguercio et al. (2009) ${ }^{\mathrm{i}}$ & Estados Unidos & 21 & Campo \\
\hline Simões $(2010)^{j}$ & Brasil & 3 & Laboratório \\
\hline \multirow[t]{2}{*}{ Bastos $(2011)^{k}$} & Brasil & 20 & Casa de vegetação \\
\hline & & 10 & Campo \\
\hline Bastos $(2012)^{1}$ & Brasil & 10 & Casa de vegetação \\
\hline Simões et al. $(2012)^{\mathrm{m}}$ & Estados Unidos & 3 & Laboratório \\
\hline
\end{tabular}

a,b,c,d,e,f,g,h,i,j,k,l,m Referências para correspondência com a Tabela 2.

Tabela 2. Eficiência de espécies/isolados de fungo no controle biológico da vassoura de bruxa do cacaueiro com base na literatura, onde I-

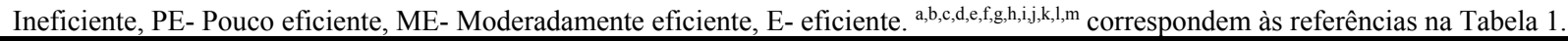

\begin{tabular}{|c|c|c|}
\hline Espécie/isolado & Eficiência (\%) & Classificação ${ }^{1}$ \\
\hline Botryosphaeria ribis Grossenb. \& Duggar ${ }^{\mathrm{h}}$ & 27,00 & PE \\
\hline Clonostachys rosea (Link) Schroers, Samuels, Seifert \& W. Gams ${ }^{\text {h }}$ & 27,00 & $\mathrm{PE}$ \\
\hline Clonostachys sp. ${ }^{\mathrm{a}}$ & 16,70 & I \\
\hline Clonostachys sp. ${ }^{\mathrm{b}}$ & $35,00^{2}$ & $\mathrm{PE}$ \\
\hline Clonostachys sp. ${ }^{\mathrm{k}}$ & $41,73^{3}$ & PE \\
\hline Clonostachys sp. ${ }^{\mathrm{c}}$ & 70.84 & ME \\
\hline Colletotrichum gloeosporioides (Penz.) Penz. \& Sacc. ${ }^{\mathrm{h}}$ & 27,00 & PE \\
\hline Trichoderma atroviride Bissett $\left(\right.$ Tat2076) ${ }^{\mathrm{m}}$ & 64,24 & ME \\
\hline Trichoderma atroviride $\left(\right.$ Tat2076) ${ }^{\mathrm{j}}$ & 54,22 & ME \\
\hline Trichoderma brevicompactum G.F. Kraus, C.P. Kubicek \& W. Gams ${ }^{1}$ & 12,00 & I \\
\hline Trichoderma brevicompactum ${ }^{\mathrm{g}}$ & 40,00 & $\mathrm{PE}$ \\
\hline Trichoderma harzianum Rifai (Th1058) ${ }^{\mathrm{j}}$ & 47,52 & PE \\
\hline Trichoderma harzianum $(\mathrm{Th} 1070)^{\mathrm{j}}$ & 59,68 & ME \\
\hline Trichoderma harzianum $\left(\right.$ Th1070) ${ }^{\mathrm{m}}$ & 59,87 & ME \\
\hline Trichoderma harzianum $(\mathrm{Th} 906)^{\mathrm{j}}$ & 51,31 & ME \\
\hline Trichoderma harzianum $\left(\right.$ Th911) ${ }^{\mathrm{j}}$ & 90,33 & $\mathrm{E}$ \\
\hline Trichoderma harzianum $(\mathrm{Th} 911)^{\mathrm{m}}$ & 91,86 & $\mathrm{E}$ \\
\hline Trichoderma longibrachiatum Rifai $(\operatorname{Tlg} 3188)^{\mathrm{j}}$ & 45,20 & PE \\
\hline Trichoderma longibrachiatum $(\mathrm{Tlg} 3188)^{\mathrm{m}}$ & 57,84 & ME \\
\hline Trichoderma pilulliferum Webster \& Rifai (Tp1925) & 60,68 & ME \\
\hline Trichoderma pilulliferum $(\mathrm{Tp} 1925)^{\mathrm{m}}$ & 68,71 & ME \\
\hline Trichoderma pseudokoningii Rifai $(\operatorname{Tps} 1052)^{\mathrm{j}}$ & 54,41 & ME \\
\hline Trichoderma pseudokoningii $(\operatorname{Tps} 1541)^{\mathrm{j}}$ & 42,38 & PE \\
\hline Trichoderma pseudokoningii $(\operatorname{Tps} 907)^{\mathrm{j}}$ & 39,82 & $\mathrm{PE}$ \\
\hline Trichoderma pseudokoningii $(\mathrm{Tps} 909)^{\mathrm{j}}$ & 41,51 & PE \\
\hline Trichoderma pseudokoningii $(\mathrm{Tps} 913)^{\mathrm{j}}$ & 50,25 & PE \\
\hline Trichoderma pseudokoningii $(\operatorname{Tps} 1052)^{\mathrm{m}}$ & 73,72 & ME \\
\hline Trichoderma reesei E.G. Simmons $(\operatorname{Tr} 1612)^{j}$ & 22,12 & $\mathrm{PE}$ \\
\hline Trichoderma reesei $(\operatorname{Tr} 1612)^{\mathrm{m}}$ & 24,17 & I \\
\hline Trichoderma stromaticum Samuels \& Pardo-Schulth ${ }^{\mathrm{e}}$ & $44,91^{4}$ & ME \\
\hline Trichoderma stromaticum ${ }^{\mathrm{f}}$ & 46,60 & $\mathrm{PE}$ \\
\hline Trichoderma stromaticum ${ }^{\mathrm{i}}$ & 55,20 & ME \\
\hline Trichoderma stromaticum ${ }^{\mathrm{d}}$ & 80,90 & ME \\
\hline Trichoderma stromaticum $(\mathrm{Ts} 3768)^{\mathrm{j}}$ & 42,93 & PE \\
\hline Trichoderma stromaticum $(\mathrm{Ts} 1441)^{\mathrm{j}}$ & 39,63 & PE \\
\hline Trichoderma stromaticum $(\mathrm{Ts} 1445)^{\mathrm{j}}$ & 40,73 & $\mathrm{PE}$ \\
\hline
\end{tabular}


Tabela 2 (continuação)

\begin{tabular}{|c|c|c|}
\hline Espécie/isolado & Eficiência $(\%)$ & Classificação $^{1}$ \\
\hline Trichoderma stromaticum $(\mathrm{Ts} 2994)^{\mathrm{j}}$ & 37,71 & $\mathrm{PE}$ \\
\hline Trichoderma stromaticum $(\mathrm{Ts} 3109)^{\mathrm{j}}$ & 40,47 & PE \\
\hline Trichoderma stromaticum $(\mathrm{Ts} 3454)^{\mathrm{j}}$ & 44,93 & $\mathrm{PE}$ \\
\hline Trichoderma stromaticum $(\mathrm{Ts} 3461)^{\mathrm{j}}$ & 27,78 & PE \\
\hline Trichoderma stromaticum $(\mathrm{Ts} 4077)^{\mathrm{j}}$ & 41,43 & $\mathrm{PE}$ \\
\hline Trichoderma stromaticum $(\operatorname{Ts} 3454)^{\mathrm{m}}$ & 47,72 & ME \\
\hline Trichoderma virens J.H. Mill., Giddens \& A.A.Foster (Tvi2007) & 54,98 & ME \\
\hline Trichoderma virens Persoon (Tvi2007) ${ }^{\mathrm{m}}$ & 62,58 & ME \\
\hline Trichoderma viride $(\operatorname{Tvr} 1643)^{\mathrm{j}}$ & 56,81 & ME \\
\hline Trichoderma viride (Tvr905) ${ }^{\mathrm{j}}$ & 74,85 & ME \\
\hline Trichoderma viride $(\mathrm{Tvr} 905)^{\mathrm{m}}$ & 74,67 & $\mathrm{ME}$ \\
\hline Trichothecium roseum (Pers.) ${ }^{\mathrm{g}}$ & 28,00 & PE \\
\hline Fungos endofíticos ${ }^{5 c}$ & 16,22 & I \\
\hline
\end{tabular}

${ }^{1}$ Classificação segundo Linhares et al. (1995)

${ }^{2}$ Média entre dois tratamentos (Aplicação no tronco e aplicação na folhagem)

${ }^{3}$ Média entre dois tratamentos (Casa de vegetação e campo)

${ }^{4}$ Média entre dois tratamentos (Estação chuvosa e estação seca)

${ }^{5}$ Acremonium sp., Blastomyces sp., Botryosphaeria sp., Cladosporium sp., Colletotrichum gloeosporiodes Penz., Cordyceps sobolifera (Hill) Berk. \& Broome, Diaporthe phaseolorum Cooke \& Ellis, D. helianthi Munt.-Cvetk., Mihaljc. \& M. Petrov, Fusarium sp., F. chlamydosporum Wollenw. \& Reinking, F. oxysporum Schlecht., F. polyphialidicum Marasas, P.E. Nelson, Toussoun \& P.S. van Wyk, Geotrichum sp., Gibberella zeae (Schwein.) Petch [teleom.], G. fujikuroi (Sawda) Wollenweber, G. moniliformis Wineland, Gliocladium sp., G. catenulatum J.C. Gilman \& E.V. Abbott, Lasiodiplodia theobromae (Pat.) Griffon \& Maubl., Monilochoetes sp., Nectria haematococca Berk. \& Broome, Pestalotiopsis microspora (Speg.) Bat. \& Peres, Phomopsis sp., Pleurotus ostreatus (Jacq.) P. Kumm., Pseudofusarium purpureum Matsush., Rhizopycnis vagum D.F. Farr, Syncephalastrum sp., Trichoderma sp., Verticillium sp., V. luteo-album (Link) Subram., Xylaria sp.)

seguida pelas condições de campo $(46,10 \%)$ e casa de vegetação (35\%). Entretanto, essa diferença é estatisticamente significativa apenas para os resultados obtidos em laboratório e em casa de vegetação $(\mathrm{p}=$ 0.0473) (Figura 2)

\section{DISCUSSÃo}

A eficiência do gênero Trichoderma no controle biológico tem sido amplamente registrada na literatura (Saito et al. 2009). Segundo Menezes et al. (2010), fungos desse gênero são naturalmente encontrados no solo e apresentam uma importante função ecológica,

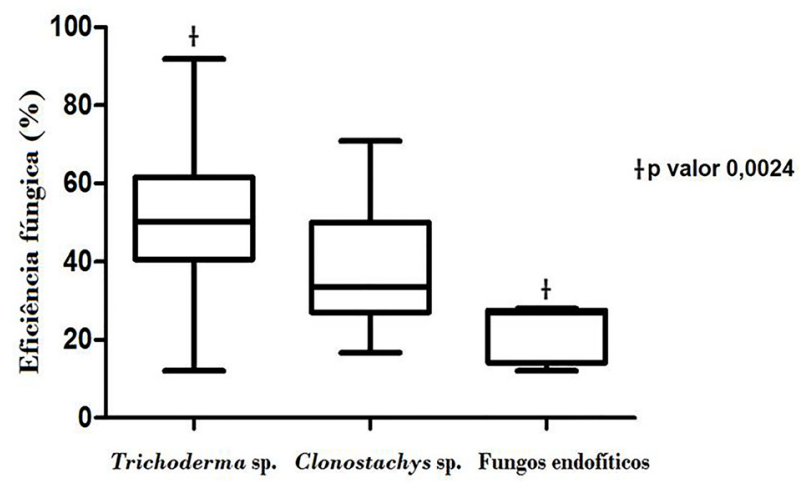

Figura 1. Agrupamento das espécies fúngicas em relação à eficiência no controle biológico da vassoura de bruxa do cacaueiro. pois participam da decomposição e mineralização dos resíduos vegetais, contribuindo com a disponibilização de nutrientes para as plantas. O gênero Trichoderma apresenta características essenciais para um agente de controle biológico: 1- ausência de impacto negativo ao meio ambiente; 2- presença de estruturas de reprodução de fácil propagação, principalmente em substratos naturais; 3- capacidade de sobreviver em ambientes desfavoráveis; 4- além de conter populações de patógenos em condições de solo diferentes (Vinale et al. 2008).

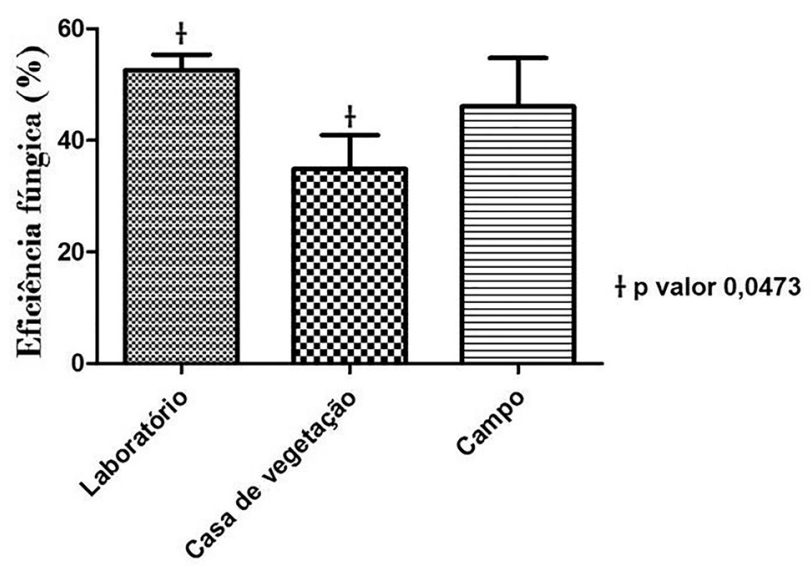

Figura 2. Efeito das condições de experimento (laboratório, casa de vegetação e campo) sobre a eficiência do agente biocontrolador da doença vassoura de bruxa em cacaueiro. 
A eficiência dos isolados de Trichoderma harzianum e T. stromaticum se deve, provavelmente, aos seus mecanismos de competitividade, de antibiose e de micoparasitismo, agindo sinergicamente no hospedeiro. Segundo Sanogo et al. (2002), os microorganismos antagonistas devem competir por espaço e nutrientes com o fitopatógeno. Portanto, é importante que o fungo utilizado em biocontrole apresente uma boa velocidade de colonização, o que determina a sua habilidade em se estabelecer no substrato-alvo. Embora o gênero Trichoderma tenha apresentado uma média de eficiência no biocontrole da vassoura de bruxa de $51 \%$, seus isolados apresentam variações quanto à eficiência. Isto pode estar relacionado à especificidade entre alguns antagonistas e o fitopatógeno, podendo haver o envolvimento de vários genes e de outros fatores genéticos que interagem com o ambiente (Bastos 2007).

Espécies de Trichoderma podem ainda inibir ou matar fungos fitopatogênicos através da produção de antibióticos antifúngicos e/ou enzimas hidrolíticas. A capacidade de produzir enzimas líticas, como quitinases, glucanases e proteases, está diretamente envolvida no processo de micoparasitismo que favorece espécies deste gênero a funcionarem como agentes no controle biológico de fitopatógenos (Suarez et al. 2004; Almança 2008; Santin 2008).

Fungos do gênero Clonostachys também têm sido relatados como agentes eficientes no biocontrole da vassoura de bruxa, principalmente por apresentarem habilidade para crescer endofiticamente em tecidos verdes sem causar danos ao hospedeiro. Esta habilidade confere ao antagonista uma vantagem competitiva importante em relação ao patógeno quanto à colonização dos tecidos vivos (Sutton et al. 1997). Segundo Bastos (2011), a atividade de controle exercida por Clonostachys pode ser atribuída a determinada(s) substância(s) produzida(s) por este gênero ou através da indução de resistência que impede o desenvolvimento do patógeno. Colonizando o interior da planta hospedeira, a espécie pode competir por espaço e nutriente com o patógeno, reduzindo o aparecimento da doença. Contudo, a indução de resistência sistêmica parece ser o mecanismo mais importante para o controle das doenças, embora a produção de compostos antagônicos também possa estar envolvida. Existem ainda espécies de Clonostachys que induzem a resistência do hospedeiro.

A eficiência média das espécies de fungos no controle da vassoura de bruxa foi aproximadamente $50 \%$. Ou seja, a severidade da fitopatogenicidade foi reduzida pela metade. De modo geral, o micoparasitismo parece ser o mecanismo mais eficiente de antagonismo no controle biológico. No entanto, Bastos (2012) ressaltou que o biocontrole pode reduzir a incidência da doença, mas não elimina completamente o patógeno. No manejo integrado, misturas de antagonistas com diferentes modos de ação poderiam ser compatibilizadas (Ozbay \& Newman 2004). Assim, a ação das espécies de Trichoderma e Clonostachys poderiam atuar de maneira complementar no controle da doença. Outra forma seria a aplicação do antagonista em combinação com cultivares resistentes a doenças e práticas de saneamento.

Canci et al. (1997), Soares et al. (2006) e Brand et al. (2009) sugeriram que experimentos realizados em campo são susceptíveis a diferenças no relevo, na vegetação e no solo. Em casa de vegetação, são observadas diferenças na incidência luminosa, na temperatura, na irrigação e na ventilação. Em laboratório, sob condições totalmente controladas, os testes são mais impactantes (Potrich et al. 2009). Além disso, no campo, os micro-organismos necessitam de um tempo mínimo para se adaptarem às novas condições ambientais. Essa adaptação torna a ação microbiana mais lenta, o que deixa os agricultores desconfiados da efetividade do controle biológico (Morandi \& Bettiol 2009). Segundo Machado et al. (2012), o comportamento de Trichoderma no ambiente rizosférico pode ser diferente do comportamento in vitro devido à presença de outros micro-organismos no local e os mecanismos de ação dos micro-organismos podem variar conforme a linhagem microbiana e o ambiente, sendo suscetíveis à interferência de outros micro-organismos, substrato, temperatura e umidade.

Os benefícios do controle biológico são muitos, especialmente no que se refere à redução do uso de insumos químicos como agrotóxicos em culturas agrícolas. $\mathrm{O}$ controle químico propicia alta produtividade, mas seus efeitos negativos (toxicidade) sobre o meio ambiente devem ser considerados, e mutantes resistentes aos princípios ativos podem ser selecionados (Morandi \& Bettiol 2009). São geralmente necessárias repetidas aplicações, lançando grandes quantidades de produtos tóxicos no ambiente, e o custo associado a essa técnica é alto (Melo \& Costa 2005). O tempo de degradação dessas substâncias é lento e elas permanecem em concentrações elevadas na cadeia alimentar.

O controle biológico é uma alternativa viável em programas de manejo integrado da vassoura de bruxa do cacaueiro, especialmente quando os agentes do biocontrole forem espécies fúngicas dos gêneros Trichoderma e Clonostachys. Apesar das vantagens e benefícios do gênero Trichoderma no controle biológico, há poucas formulações à base deste microorganismo devidamente registradas pelo MAPA (Ministério da Agricultura, Pecuária e Abastecimento) no Brasil (Machado et al. 2012). De acordo com Melo $\&$ Costa (2005), isto representa um fator limitante para o biocontrole na agricultura, uma vez que os fabricantes são submetidos aos mesmos critérios que regulamentam o registro de agrotóxicos, tratando-se de um processo oneroso e realizado em um longo período de tempo. 
O número de artigos abordando o biocontrole em diversas fitopatogenicidades tem crescido nas últimas décadas. No entanto, os resultados em relação à eficiência deste método ainda apresentam lacunas, especialmente no que tange à relação entre condições experimentais, agente biocontrolador, agente patogênico e o cultivar agrícola.

\section{AGRADECIMENTOS}

Os autores agradecem à Universidade Federal da Bahia pelo apoio e ao Professor Antônio Carlos Ricardo Braga Júnior pela colaboração nas análises estatísticas.

\section{REFERÊNCIAS}

Almança, M.A.K. 2008. Aspectos da Interação Arroz-Trichoderma spp. em Solos Alagados. Dissertação de Doutorado. Universidade Federal do Rio Grande do Sul.

Alves, S.A.M.; Pomella, A.W.V.; Aitken, W.M. \& Bergamin Filho, A. 2006. Curvas de progresso e gradientes da vassourade-bruxa (Crinipellis perniciosa) em cacaueiros enxertados em Uruçuca, Bahia. Fitopatologia Brasileira 31(5): 483-491.

Bastos, C.N. 2004. Biocontrole da vassoura-de-bruxa do cacaueiro pelo uso do Clonostachys sp. Fitopatologia Brasileira 29: 37 38.

Bastos, C.N. 2007. Produção de Metabólitos de Trichothecium roseum e seu Efeito no Controle da Vassoura-de-Bruxa do Cacaueiro. Fundag/Embrapa, Campinas.

Bastos, C.N. 2008 Método para preservação da viabilidade e atividade antagônica de Trichoderma stromaticum, agente de biocontrole da vassoura-de-bruxa do cacaueiro. Summa Phytopathologica 34(3): 265-266.

Bastos, C.N. 2011. Avaliação do potencial de Clonostachys sp. no biocontrole da vassoura-de-bruxa do cacaueiro. Agrotrópica 23(2): 95-100.

Bastos, C.N. 2012. Isolate of Trichoderma brevicompactum for the control of cocoa witches' broom disease: preliminary results. Agrotrópica 24(1): 5-10.

Borges, G.F.; Teixeira, A.M. \& Ferreira, J.P. 2012. Meta-análise do efeito no sistema imunitário da suplementação de hidratos de carbono no exercício físico. Motricidade 8(2): 83-97.

Brand, S.C.; Antonello, L.M.; Muniz, M.F.B.; Blume, E.; Santos, V.J. \& Reiniger, L.R.S. 2009. Qualidade sanitária e fisiológica de sementes de soja submetidas a tratamento com bioprotetor e fungicida. Revista Brasileira de Sementes 31(4): 87-94.

Campos, Â.D.; Hampe, M.M.V.; Ferreira, A.G.; Antunes, I.F. \& Castro, L.D. 2009. Indução de resistência sistêmica à antracnose em feijoeiro-comum pela raça delta avirulenta de Colletotrichum lindemuthianu. Pesquisa Agropecuária Brasileira 44(1): 15-21.

Canci, P.C.; Carvalho, F.I.F.; Neto, J.F.B.; Oliveira, M.A.R. \& Franco, F.A. 1997. Diferentes ambientes para avaliação da sensibilidade ao ácido giberélico em genótipos de trigo (Triticum aestivum L.). Ciência Rural 27(1): 21-25.
Carvalho, A.C. 2006. Bioprospecção de Isolados de Trichoderma stromaticum para o Controle Biológico da Vassoura-de-Bruxa do Cacaueiro. Dissertação de Mestrado. Universidade Estadual de Santa Cruz.

Costa, J.C.B.; Bezerra, J.L.; Santos Filho, L.P.; Alves, M.C. \& Moura, E.M. 2009. Controle biológico da vassoura-de-bruxa do cacaueiro da Bahia, Brasil. In: W. Bettiol, \& M.A.B. Morandi (orgs), Biocontrole de Doenças de Plantas: uso e perspectivas. Embrapa Meio Ambiente, Jaguariúna, p. 245-266.

Krauss, U. \& Soberanis, W. 2001. Biocontrol of cocoa pod diseases with mycoparasite mixtures. Biological Control 22: 149-158.

Linhares, A.I.; Matsumura, A. \& Luz, V. 1995. Avaliação da amplitude de ação antagonística de microrganismos epífitas do trigo sobre o crescimento radial de Drechslera tritici-repentis. Revista Brasileira de Agrociência 1(3): 119-126.

Loguercio, L.L.; Carvalho, A.C.; Niella, G.R.; Souza J.T. \& Pomella, A.W.V. 2009. Selection of Trichoderma stromaticum isolates for efficient biological control of witches' broom disease in cacao. Biological Control 51: 130-139.

Machado, D.F.M.; Parzianello, F.R.; Silva, A.C.F. \& Antoniolli, Z.I. (2012). Trichoderma no Brasil: o fungo e o bioagente. Revista de Ciências Agrárias 35(1): 274-288.

Meinhardt, L.W.; Rincones, J.; Bailey, B.A.; Aime, M.C.; Griffith, G.W.; Zhang, D. \& Pereira, G.A. 2008. Moniliophthora perniciosa, the causal agent of witches' broom disease of cacao: what's new from this old foe? Molecular Plant Pathology 9(5): 577-588.

Mejía, L.C.; Rojas, E.I.; Maynard, Z.; Bael, S.V.; Arnold, A.E.; Hebbar, P.; Samuels, G.J.; Robbins, N. \& Herre, E.A. 2008. Endophytic fungi as biocontrol agents of Theobroma cacao pathogens. Biological Control 46: 4-14.

Melo, I.S. \& Costa F.G. 2005. Desenvolvimento de uma formulação granulada a base de Trichoderma harzianum para o controle de fitopatógenos. Comunicado Técnico Embrapa 31: $1-4$.

Menezes, J.P.; Junges, E.; Blume, E. \& Pereira, M.E. 2010. Toxicologia do biopreparado a base de Trichoderma sp. (isolado UFSM T17) administrado em mamífero. Revista da FZVA 17(1): 38-50.

Morandi, M.A.B. \& Bettiol, W. 2009. Controle biológico de doenças de plantas no Brasil. In: W. Bettiol \& M.A.B. Morandi (orgs), Biocontrole de Doenças de Plantas: uso e perspectivas. Embrapa Meio Ambiente, Jaguariúna, p. 7-14.

Ozbay, N. \& Newman, S.E. 2004. Biological control with Trichoderma spp. with emphasis on T. harzianum. Pak Journal Biological Science 7(4): 478-484.

Pelozatto, D. \& Fernandes, R.A. 2011. Relação entre qualidade de vida e atividade física: uma revisão sistemática da literatura nacional. Colloquium Vitae 3(2): 54-58.

Potrich, M.; Alves, L.A.; Haas, J.; Silva, E.L.; Daros, A.; Pietrowsk, V. \& Neves, P.M.O.J. 2009. Seletividade de Beauveria bassiana e Metarhizium anisopliae a Trichogramma pretiosum Riley (Hymenoptera: Trichogrammatidae). Neotropical Entomology 38(6): 822-826.

Rubini, M.R.; Silva-Ribeiro, R.T.; Pomella, A.W.V.; Maki, C.S.; Araújo, W.L.; Santos, D.R. \& Azevedo, J.L. 2005. Diversity of endophytic fungal community of cacao (Theobroma cacao 
L.) and biological control of Crinipellis perniciosa, causal agent of witches' broom disease. International Journal of Biological Sciences 1: 24-33.

Saito, L.R.; Sales, L.L.S.R.; Martinckoski, L.; Royer, R.; Ramos, M.S. \& Reffatti, T. 2009. Aspects of the effects of the fungus Trichoderma spp. in biocontrol of pathogens of agricultural crops. Pesquisa Aplicada \& Agrotecnologia 2(3): 203-208.

Samuels, G.J. 2006. Trichoderma: systematics, the sexual state, and ecology. Phytopatology 96(2): 195-206.

Sanogo, S.; Pomella, A.; Hebbar, P.K.; Bailey, B.; Costa, J.C.B.; Samuels, G.J. \& Lumsden, R.D. 2002. Production and germination of conidia of Trichoderma stromaticum, a mycoparasite of Crinipellis perniciosa on cacao. Phytopathology 92(10): 1032-1037.

Santin, R.C.M. 2008. Potencial do Uso dos Fungos Trichoderma spp. e Paeciolmyces lilacinus no Biocontrole de Meloidogyne incognita em Phaseolus vulgaris. Dissertação de Doutorado. Universidade Federal do Rio Grande do Sul.

Santos, L.S. 2005. Interação Antagônica Trichoderma stromaticum - Crinipellis perniciosa: efeito do clima no biocontrole da vassoura de bruxa do cacaueiro e desenvolvimento de marcadores microssatélites. Dissertação de Mestrado. Universidade Estadual de Santa Cruz.

Schrank, A.; Franceschini, M.; Guimarães, A.P.; Camassola, M.; Frazzon, A.P.; Baratto, C.M.; Kogler, V.; Silva, M.V.; Dutra, V.; Nakazoto, L.; Castro, L.; Santi, L. \& Vainstein, M.H. 2001. Biotecnologia aplicada ao controle biológico. Biotecnologia Ciência \& Desenvolvimento 23: 32-37.

Simões, M.L.G. 2010. Controle Biológico de Moniliophthora perniciosa, Agente Causal da Vassoura de Bruxa do Cacaueiro, por Diferentes Espécies e Linhagens de Trichoderma spp. Dissertação de Doutorado. Universidade Estadual Paulista.

Simões, M.L.G.; Tauk-Tornisielo, S.M.; Niella, G.R. \& Tapia, D.M.T. 2011. Evaluation of Trichoderma spp. for the biocontrol of Moniliophthora perniciosa subgroup 1441. Journal of Biology and Life Science 3(1): 18-36.

Soares A.L.L.; Pereira, J.P.A.R.; Ferreira, P.A.A.; Vale, H.M.M.; Lima, A.S.; Andrade, M.J.B. \& Moreira, F.M.S. 2006. Eficiência agronômica de rizóbios selecionados e diversidade de populações nativas nodulíferas em perdões (MG). I-Caupi. Revista Brasileira de Ciência do Solo 5: 795802.

Souza, J.T.; Pomella, A.W.V.; Bowers, J.H.; Pirovani, C.P.; Loguercio, L.L. \& Hebbar, K.P. 2006. Genetic and biological diversity of Trichoderma stromaticum, a mycoparasite of the cacao witches'-broom pathogen. The American Phytopathological Society 96(1): 61-67.

Suarez, B.; Rey, M.; Castillo, P.; Monte, E. \& Llobell, A. 2004. Isolation and characterization of PRA1, a trypsin-like protease from the biocontrol agent Trichoderma harzianum CECT 2413 displaying nematicidal activity. Applied Microbiology and Biotechnology 65(1): 46-55.

Sutton, J.C.; Li, D.W.; Peng, G.; Yu, H.; Zhang, P. \& Valdebenito-Sanhueza, R.M. 1997. Gliocladium roseum a versatile adversary of Botrytis cinerea in crops. Plant Disease 81(4): 316-328.

Vinale, F.; Sivasithamparam, K.; Ghisalberti, E.L.; Marra, R.; Barbetti, M.J.; Li, H.; Woo, S.L. \& Lorito, M. 2008. A novel role for Trichoderma secondary metabolites in the interactions with plants. Physiological and Molecular Plant Pathology 72(1): 80-86. 